\title{
Nodular Lymphocyte Predominant Hodgkin's Lymphoma with T Cell/ Histiocyte Rich Large B Cell Lymphoma Like Transformation
}

\author{
Sakshi Batra, Harsh Batra, Charanjeet Ahluwalia and Sunil Ranga \\ Department of Pathology and Laboratory Medicine, VMMC and Safdarjung Hospital, Delhi, India
}

\section{ABSTRACT}

Nodular lymphocyte predominant Hodgkin's lymphoma (NLPHL) is an uncommon variant form of Hodgkin's lymphoma with a worldwide incidence of 5\%. It has long been recognized that NLPHL can have varied growth patterns, including some with diffuse areas and/or numerous T cells. NLPHL may evolve to a completely diffuse T-cell-rich proliferation lacking any follicular dendritic cells which would be consistent with a T-cell histiocyte-rich large B-cell lymphoma (THRLBCL) or can be associated with such a proliferation at a separate site. Recent data indicate that progression to a process with features of THRLBCL is associated with a more aggressive clinical course, and requires different management, hence the significance of diagnosing correctly THRLBCL-like transformation of NLPHL. We report a case of THRLBCL-like transformation of NLPHL in an 11-year old male child.

Keywords: T-cell histiocyte-rich large B-cell lymphoma, Lymphoma, Hodgkin's Lymphoma, LP cells, Immunohistochemistry

\section{Introduction}

Nodular lymphocyte predominant Hodgkin's lymphoma (NLPHL) is an uncommon variant form of Hodgkin's lymphoma with a worldwide incidence of $5 \% .{ }^{1}$ It is characterized by proliferation of scattered large neoplastic cells (lymphocytic and/or histiocytic [L\&H] cells or "popcorn" cells) in large spherical meshwork of follicular dendritic cells filled with non-neoplastic small lymphocytes, mainly of B-cell type. It has long been recognized that NLPHL can have varied growth patterns, including some with diffuse areas and/or numerous $\mathrm{T}$ cells. ${ }^{2}$ NLPHL may evolve to a completely diffuse T-cellrich proliferation lacking any follicular dendritic cells which would be consistent with a T-cell histiocyte-rich large B-cell lymphoma (THRLBCL) or can be associated with such a proliferation at a separate site. We report a case of THRLBCL-like transformation of NLPHL in an 11-year old male child.

\section{Case Report}

An 11-year old male child presented in the paediatrics OPD with complaint of vomiting for 4-5 consecutive days for one month and swelling in left side of neck for two years. Patient gave a history of generalised weakness and on-off history of fever. However, no history of significant weight loss, night sweats, body pain or abdominal swelling or fullness was present. Past history was not significant. Family history was not significant. On general physical examination, vitals were within normal limits without cyanosis, clubbing or icterus. Local examination revealed multiple enlarged palpable lymph nodes measuring
$1-1.5 \mathrm{~cm}$ in size in posterior triangle of left sided cervical region. Excision biopsy of cervical lymph node was done and sent to the department of pathology. Histopathological examination of the biopsy revealed a lymph node whose architecture was effaced by ill-defined nodules of small lymphocytes and macrophages with presence of large cells located within these nodules (fig.1). These large cells had abundant eosinophilic cytoplasm with round to oval nucleus, vesicular chromatin and prominent eosinophilic nucleoli. Some of these cells were multi-nucleated, while some had irregular nuclear outlines. Occasional mitotic figures were seen (fig.2). These large cells were positive for LCA, PAX-5 and CD-20 and negative for CD-15, CD30 , ALK and Bcl-6, suggesting them to be lymphocyte predominant (LP) cells. The predominant population of background lymphocytes were positive for CD-3 and CD-5, rest of the small lymphocytes were positive for CD-19 and CD-20 (fig.3). Also seen were histiocytes in the background admixed with the small lymphocytes which were positive for CD-68. Hence considering these histomorphological and immunohistochemical features, diagnosis of nodular lymphocyte predominant Hodgkin's lymphoma with T-cell/histiocyte rich large B-cell lymphoma like transformation was rendered.

\section{Discussion}

Nodular lymphocyte predominant Hodgkin's lymphoma (NLPHL) is a germinal centre (GC) B-cell derived neoplasm preferentially affecting young to middle aged male patients., ${ }^{3,4}$ Diagnosis of NLPHL often reveals a limited stage disease with an indolent clinical 


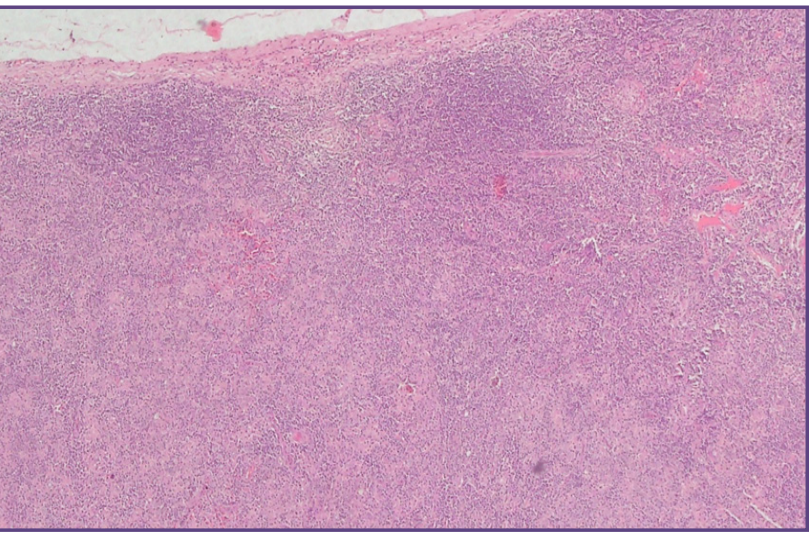

Fig. 1: Effaced lymph node architecture with ill defined nodules composed of small lymphocytes and macrophages.

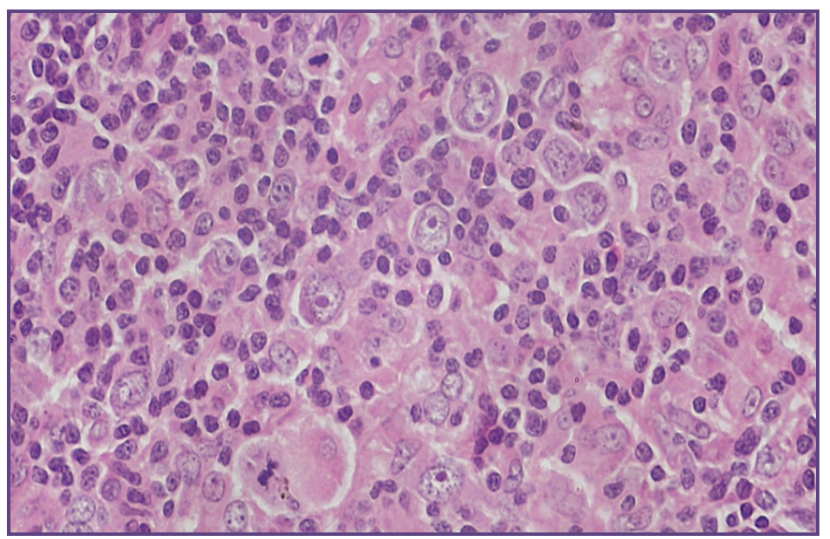

Fig. 2: Lymphocyte predominant (LP) cells admixed with background lymphocytes and macrophages. Atypical mitotic figures also noted.
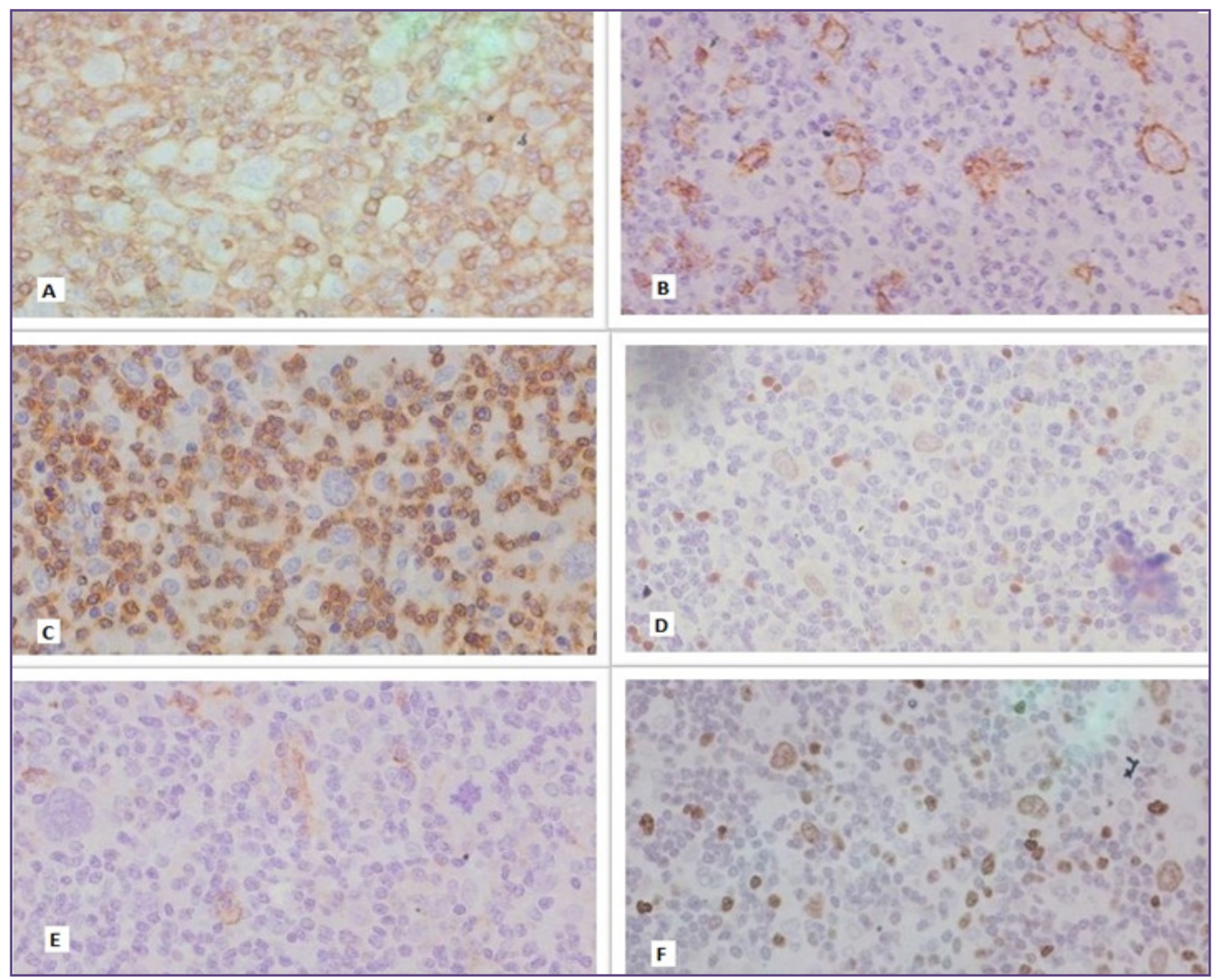

Fig. 3: IHC Markers Peformed A. LCA, B. CD20, C. CD3, D. PAX5, E. EMA, F. Ki-67. 
behaviour. ${ }^{5}$ In most cases the histopathologic picture of NLPHL is dominated by a nodular infiltrate composed of reactive small $\mathrm{B}$ lymphocytes and the blastic tumour cells, termed lymphocyte predominant (LP) cells, are located within these nodules. LP cells are derived from germinal centre B cells, show ongoing somatic hypermutation, 3,4,6 and express B-cell markers at varying intensities. ${ }^{7,8,9}$ However, cases of NLPHL have been described showing a diffuse infiltrate of LP cells in a T cell and histiocyte-rich background. ${ }^{2,10}$ Six NLPHL variant patterns have been defined by rephrase as" Fan et al viz classic (B-Cell-rich) nodular, serpiginous/interconnected nodular, nodular with prominent extranodular L\&H cells, T-cell-rich nodular, diffuse with a T-cell/histiocyte rich background (T-cell/histiocyte rich B-cell lymphoma [THRLBCL]-like), and a (diffuse) B-cell-rich pattern. ${ }^{2}$ In their study, Fan et al followed up 56 patients of NLPHL and found that the presence of a diffuse (THRLBCL-like) pattern was significantly more common in patients with recurrent disease $(30 / 56)$ than those without recurrence $(26 / 56)$; and the presence of a diffuse pattern (THRLBCLlike) was shown to be an independent predictor of recurrent disease $(\mathrm{P}=0.00324) .{ }^{2}$ In addition, there is a tendency for progression to an increasingly more diffuse pattern over time. Analysis of sequential biopsies from patients with recurrent disease suggests that the presence of prominent extranodular L\&H cells might represent early evolution to a diffuse (THCRBCL-like) pattern. $60 \%$ of these rare THRLBCL-like NLPHL cases present with advanced clinical stages (III/IV). ${ }^{10}$

In our case, the tumour cells were large and pleomorphic with irregular nuclear contours showing multilobation with prominent eosinophilic nucleoli, resembling popcorn cells or lymphocyte predominant (LP) variant of Reed Sternberg cell. These tumour cells were positive for PAX-5 and CD20, confirming their B-cell origin. However, they were negative for CD-15 and CD-30, ruling out the possibility of classical Hodgkin's Lymphoma. These cells were negative for EMA and Bcl-2, ruling out the possibility of anaplastic large cell lymphoma and T-cell/histiocyte rich large B-cell lymphoma respectively. The predominant population of background lymphocytes was positive for CD-3 and CD-5 with a significant number of histiocytes (CD-68 positive). This finding of T-cell/histiocyte rich background with LP cells is unusual for commonly occurring classic nodular or diffuse variant of NLPHL. 'THRLBCL-like transformation of NLPHL' is the new terminology proposed for the variant of NLPHL characterised by diffuse arrangement of background lymphocytes predominantly comprised of T-cells and a few B-cells.

Both the diseases- NLPHL and THRLBCL contain neoplastic cells with similar morphologic and immunophenotypic features but differ with respect to their architecture and the nature of the reactive background. Because of the morphologic and immunophenotypic similarities between NLPHL and THRLBCL, it is possible that these two entities may represent different stages of the same disease. A possible biologic relation of THRLBCL with NLPHL has been suggested. In fact, a biologic continuum has been supported by gene expression profiling studies that have demonstrated a surprisingly high similarity of LP cells to the tumour cells of THRLBCL and classic Hodgkin's lymphoma. ${ }^{11}$ Comparative Genomic Hybridization studies have revealed a few overlapping recurrent genetic abnormalities in NLPHL and THRLBCL. These overlapping abnormalities might be the genetic link between NLPHL and THRBCL. ${ }^{12,13}$ The observation that NLPHL can show a THRLBCL-like transformation, which is indistinguishable from primary THRLBCL, further reiterates the link between the two entities. It is likely that the distinction between NLPHL and THRLBCL does not lie in different genomic alterations and the immunophenotype of the tumour cells, but rather in the different cellular composition of the microenvironment as described above.

\section{Conclusion}

Recent data indicate that progression to a process with features of THRLBCL is associated with a more aggressive clinical course, and requires different management, such that the term NLPHL in this setting may not be sufficient. ${ }^{14}$ The 2016 revision of WHO classification of lymphoid neoplasms will recommend the designation of THRLBCLlike transformation of NLPHL. ${ }^{15}$ The present case report of THRLBCL-like transformation of NLPHL in a 11-year old child will add to the world literature on this rare entity emphasizing the significance of identifying not just the tumour cells but also the microenvironment comprised by the lymphocytes in the background in a case of lymphoma.

\section{Acknowledgements}

None

\section{Funding}

None

\section{Competing Interests}

None

\section{Reference}

1. Swerdlow SH, International Agency for Research on Cancer, World Health Organization (2008) WHO classification of tumours of haematopoietic and lymphoid tissues. Lyon, France: International Agency for Research on Cancer. 439 p. p. 
2. Fan Z, Natkunam Y, Bair E, Tibshirani R, Warnke RA (2003) Characterization of variant patterns of nodular lymphocyte predominant hodgkin lymphoma with immunohistologic and clinical correlation. Am J Surg Pathol 27: 1346-1356.

3. Küppers R, Rajewsky K, Zhao M, Simons G, Laumann R, et al. (1994) Hodgkin disease: Hodgkin and ReedSternberg cells picked from histological sections show clonal immunoglobulin gene rearrangements and appear to be derived from B cells at various stages of development. Proc Natl Acad Sci USA 91: 10962-10966.

4. Braeuninger A, Kuppers R, Strickler JG, Wacker HH, Rajewsky K, et al. (1997) Hodgkin and Reed-Sternberg cells in lymphocyte predominant Hodgkin disease represent clonal populations of germinal center-derived tumour B cells. Proc Natl Acad Sci U S A 94: 9337-9342.

5. Diehl V, Sextro M, Franklin J, Hansmann ML, Harris N, et al. (1999) Clinical presentation, course, and prognostic factors in lymphocyte-predominant Hodgkin's disease and lymphocyte-rich classical Hodgkin's disease: report from the European Task Force on Lymphoma Project on LymphocytePredominant Hodgkin's Disease. J Clin Oncol 17: 776-783

6. Marafioti T, Hummel M, Anagnostopoulos I, et al. (1997) Origin of nodular lymphocyte-predominant Hodgkin's disease from a clonal expansion of highly mutated germinal-center B cells. N Engl J Med;337(7):453-458.

7. Steimle Grauer SA, Tinguely M, Seada L, Fellbaum C, Hansmann ML (2003) Expression patterns of transcription factors in progressively transformed germinal centers and Hodgkin lymphoma.Virchows Arch ;442(3):284-293.

8. Tedoldi S, Mottok A, Ying J, et al. (2007) Selective loss of B-cell phenotype in lymphocyte predominant Hodgkin lymphoma. J Pathol ;213(4):429-440.
9. Brune V, Tiacci E, Pfeil I, et al. (2008) Origin and pathogenesis of nodular lymphocyte-predominant Hodgkin lymphoma as revealed by global gene expression analysis. J Exp Med ;205(10):2251-2268.

10. Boudova L, Torlakovic E, Delabie J, Reimer P, Pfistner B, et al. (2003) Nodular lymphocyte-predominant Hodgkin lymphoma with nodules resembling T-cell/ histiocyte-rich B-cell lymphoma: differential diagnosis between nodular lymphocyte-predominant Hodgkin lymphoma and T-cell/histiocyte-rich B-cell lymphoma. Blood 102: 3753-3758.

11. Brune V, Tiacci E, Pfeil I, et al. Origin and pathogenesis of nodular lymphocyte-predominant Hodgkin lymphoma as revealed by global gene expression analysis. J Exp Med ;2008:2251-68.

12. Zhao FX. Nodular lymphocyte-predominant Hodgkin lymphoma or Tcell/histiocyte-rich large B-cell lymphoma: the problem in "grey zone" lymphomas. Int J Clin Exp Pathol 2008;1:300-5.

13. Rudiger T, Jaffe ES, Delsol G, et al. Workshop report on Hodgkin's disease and related diseases ('grey zone' lymphoma). Ann Oncol 1998; 9(Suppl 5):S31-8

14. Hartmann S, Döring C, Jakobus C et al. (2013) Nodular Lymphocyte Predominant Hodgkin Lymphoma and T Cell/ Histiocyte Rich Large B Cell Lymphoma - Endpoints of a Spectrum of One Disease? PLoS ONE 8(11): e78812. https://doi.org/10.1371/journal.pone.0078812

15. Arber DA, Orazi A, Hasserjian R, et al. The 2016 revision to the World Health Organization classification of myeloid neoplasms and acute leukemia. Blood. 2016;127(20):2391-2405.

*Corresponding author:

Dr. Charanjeet Ahluwalia, Mailing Address: A-15/1, Vasant Vihar, New Delhi-110057 INDIA

Phone: +91 9811175639

Email: charanjeet.ahluwalia@rediffmail.com

Financial or other Competing Interests: None. 